\title{
DISTRIBUCIÓN DE LOS COLEÓPTEROS ACUÁTICOS (INSECTA, COLEOPTERA) EN DOS CUENCAS DEL SUR DE LA P. IBÉRICA. RELACIONES CON ALGUNOS FACTORES DEL MEDIO.
}

\author{
A. Gallardo', J. Fresneda² y J. Toja' \\ 1. Dpto. de Biología Vegetal y Ecología, Facultad de Biología, Universidad de Sevilla, 41080 Sevilla, España \\ 2. Camp d'Aprenentatge "Escola de Natura de la Vall de Boi", 25527 Barruera, Lleida, España.
}

Palabras clave: Coleópteros. ríos, conductividad, cloruro, altitud, temporalidad.

Keywords: Coleoptera, rivers, conductivity, chloride, altitnde, temporality.

\begin{abstract}
DISTRIBUTION OF AQUATIC COLEOPTERA IN TWO BASINS FROM THE SOUTH OF IBERIAN PENINSULA. RELATIONSHIP WITH SOME FACTORS OF THE ENVIRONMENT.

Fauna of the extreme South of the Iberian Peninsula shows a high proportion of endemic species. Here, Guadalete and Guadaira river basins are placed. Coleoptera (larvas and adults) of the headwaters of these two basins have been studied for one annual cycle and the factores of the environment relationed with them. Salinity (measured as conductivity. sulphate and chloride), flow velocity and altitude were the main parameters that explained the species distribution. Potamonectes cerisy, Ochthebius spp, Deronectes fairmairei, Yola bicarinata, Berosus hispanicus, Laccobius atrocephalus and Laccobius sp were the most halophilic species. The taxa that shown a preference towards high altitude and low levels of salinity were Gyrinus dejeani, Hydroporus basinotatus, Hydraena capta, Elmis maugetii and Riolus subviolaceus.

Temporality of the streams was a impediment for some species to establish in. Species ables to develop in environments of both temporary or semipermanent and very saline waters were Yola bicarinata, Deronectes fairmairei, Berosus hispanicus and Laccobius $\mathrm{sp}$.
\end{abstract}

\section{INTRODUCCIÓN}

La limnofauna del extremo sur de la Península Ibérica (especialmente las montañas interiores de la provincia de Cádiz y su prolongación natural en las provincias circundantes) ha mostrado una gran diversidad (BAENA, 1983, 1985; BURMEISTER，1983; FERY \& FRESNEDA, 1988; HERNANDO \& FRESNEDA, 1987; LAGAR \& FRESNEDA, 1990 por citar algunos trabajos referentes a Heteroptera y Coleoptera). Varias especies (algunas descritas recientemente) son conocidas por presentar iin área de distribución restringida a estos territorios o, en otros casos, a través de las Montañas Béticas, pertenecientes a las Montañas Bético-Rifeñas. Se considera que estas montañas fueron un gran centro de diversificación durante el Mioceno Medio, durante el cual regresiones y transgresiones marinas fueron cambiando constantemente la línea costera. Ello llevó a la crisis Mesiniana (hace 6 millones de años), en la que tuvieron lugar importantes intercambios faunísticos con la placa tectónica africana.

Limnética, 11 (1) : 19-28 (1995)

(C) Asociación Española de Limnología, Madrid. Spain
El objetivo de este trabajo ha sido el estudio dc las especies de coleópteros que habitan las cabeceras de las cuencas de los ríos Guadalete y Guadaira. Parte de la composición faunística del río Guadalete era conocida (especialmente Hydradephaga y Palpicornia), mostrando una alta proporción de especies endémicas con una distribución Bético y Bético-Rifeña. Ambas cuencas presentan características muy peculiares, especialmente la alta salinidad encontrada en la cuenca del río Guadaira (GALLARDO \& TOJA, 1989; GALLARDO, 1991; PRENDA \& GALLARDO, 1992; GALLARDO, 1993) lo que las hace interesantes para el estudio de la distribución de sus comunidades de Coleóptera. .

\section{ÁREA DE ESTUDIO Y METODOLOGÍA}

Las cuencas de los ríos Guadaira y Guadalete son adyacentes. La del Guadaira presenta una pendiente suave y está localizada sobre materiales sedimentarios (margas principalmente), característicos de la parte occidental de la Depresión del Gua- 
dalquivir. El yeso es muy abundante y eso hace que sus aguas sean muy salinas.

La cuenca del río Guadalete presenta pendientes muy abruptas (hasta del 15,2 por ciento). Litológicamente está constituida por margas, arcillas, arenisca y calizas. Algunos manantiales salinos fluyen próximos al río principal en Algodonales, incrcmentando ligeramente la salinidad de sus aguas.

La temperatura en ambas cuencas es similar y la descarga diferente. Las dos cuencas están caracterizadas por grandes irregularidades de caudal durante y entre los años, aunque es más manifiesta en Guadaira La media anual de caudal en Guadaira es de $6 \mathrm{~m}^{3} . \mathrm{S}^{1}$, encontrándose inundaciones de hasta $2.000 \mathrm{~m}^{3} . \mathrm{S}$ '. (D.G. OBRAS HIDRAÚlICAS, 1979-1991). En Guadalete el caudal medio es dc unos $17 \mathrm{~m}^{3}$. S ', con inundaciones de hasta $1.400 \mathrm{~m}$ ' S ', (BERTOLET et al., 1989)

Para realizar el estudio se tomaron siete estaciones en la cabecera dc la cuenca del Guadaira (Salado (E1), Cuadaira en Morón (E2), Aguaderilla (E3), Guadairilla (E4), Barros (ES), Gavilán (E6) y Alcaudete (E7)) y cuatro estaciones en la cabecera de la cuenca del Guadalete (Gaidovar (E8), Guadalete en Grazalema (E9), Guadalete en Algodonales (E10) y Águila (E11)).

El criterio principal por el cual se eligieron las estaciones de muestreo fue que estuvieran libres de contaminación.

Generalmente cada estación fue maestreada cada dos meses entre Enero, de 1988 y Enero de 1989, combinando métodos cualitativos y cuantitativos. Con el fin de caracterizar las propiedades físico-químicas del agua fueron analizados 19 parámetros (GALLARDO, 1991, 1993).

\section{RESULTADOS}

La característica más importante de la cuenca del Guadaira es su alta salinidad (valores máximos de conductividad medidos en el año de $8,6 \mathrm{mS}$.cm ', y 14,6 $\mathrm{mS} . \mathrm{cm}^{-1}$, en los arroyos Salado y Barros respectivamente, aunque en un estudio previo se encontraron valores más altos (GALLARDO \& TOJA, 1989). En esta misma cuenca el arroyo Alcaudete mostró una salinidad más baja, debido a que fluye por un sustrato geológico diferente. En esta estación y en la cabecera del Guadalete no llegó a medirse 1 mS.cm ' de conductividad, excepto en Algodonales donde se midieron hasta $1,3 \mathrm{mS} . c \mathrm{~cm}$ ', debido a los manantiales salinos.

En la cuenca del Guadalete se midió una velocidad de corriente de hasta $130 \mathrm{~cm} . \mathrm{s}^{\prime}$. Todas las estaciones tuvieron agua con corriente a lo largo del año, cxcepto el Aguila que estuvo seco desde Junio hasta la finalización del muestreo. En la cuenca del Guadaira la velocidad no excedió de $60 \mathrm{~cm} . \mathrm{s}^{\prime}$, y únicamente el río principal tuvo algo de corriente durante el estiaje, mientras que los arroyos de Aguaderilla, Alcaudete y Gavilán permanecieron secos durante este período (en los dos últimos arroyos esta situación se extendió desde primavera hasta el invierno siguiente).

Se hizo un análisis de componentes principales con los ejemplares recogidos en estadío larvario, resultando que los parámctros físico-quíinicos que inostraron ser los mejores discriminantes de la distribución dc las especies fueron la salinidad (medida como coiiductividad, cloruro y sulfato), velocidad de la corriente y altitud (GALLARDO, 1991). Estos parámetros son los que mejor discriminaron a las dos cuencas estudiadas, junto con la materia en suspensión (GALLARDO \& PRENDA, 1994).

A continuación se listan las especies recogidas a lo largo del muestrco, incluyendo fecha de captura, estación de muestreo y riúmero de individuos, especificando con (L) los ejemplares encontrados en estadío larvario. También se especifican los rangos de conductividad $\left(\mathrm{Cd}\right.$, en $\left.\mathrm{mS} . \mathrm{cm}^{\prime}\right)$, cloruro $(\mathrm{Cl}$, en meq. $L^{\prime}$ ) y altitud ( $\mathrm{Al}$ ) en que cada uno de los taxa ha sido recogido $((\mathrm{L})$ : rangos de ejemplares recogidos en estadío larvario; cuando no aparece ninguna indicación se trata de adultos).

Haliplus (Neohaliplus) lineatocollis (Marshain. 1802) (12.01.88) E6: 2; (24.03.88) E5: 7 (L); (30.05.88) E9: 1 (L); (29.08.88) E5: 6, E8: 4. E9: 2 (L); (31.10.88) E1: 3 (L), E9: 3 (L); (19.01.89) E1 : 1 (L), E9: 2 (L), 1.

(L) Cd: 0.7-14.6; Cl: 0.6-205; Al: 200-380.

Cd: 0.5-10,1; CI: 0,7-137; Al: 140-660.

Aulonogyrus (s.str.) stratus (F., 1792)

(29.08.88) E2: 24.

Cd: 3,2; CI: 18,9; Al: 180 .

Gyrinus (s.str.) dejeani (Brullé, 1832)

(30.05.88) E8: 19, E9: 1; (29.08.88)E8: 2; (31.10.88) E8:

26, E9: 163; (19.01.89) E9: 1.

Cd: $0,3-0,7 ; \mathrm{Cl}: 0,7-0,9 ; \mathrm{Al}: 380-660$.

Gyrinus (s.str.) urinator (Illiger, 1807)

(30.05.88) E8: 1 .

Cd: 0,$3 ; \mathrm{Cl}: 0,9 ; \mathrm{Al}: 660$.

Orectochilus (s.str) villosus (Müller, 1776) 
(24.03.88) E8: 1 (L), E9: 1 (L); (30.05.88) E9: 1 (L); (19.01.88) E9: $2(\mathrm{~L})$.

(L) Cd: 0,2-0,8; Cl: 0,7-1,0; Al: 380-660.

Hydrovatus sp (Motschulsky).

(30.05.88) El 1: 3 (L).

(L) Cd: 0,4; CI: 1,2; Al: 360 .

Yola (s.str.) bicarinata (Latreille, 1804)

(12.01.88) E5: 34 (L); (24.03.88) E1: 1 (L), E5: 9, E1 1: 5

(L); (30.05.88) E1; 8 (L), E8: 1(L), El 1: 1 (L); (29.08.88)

E9: 1 (L); (19.01.89) E9: 3 (L).

(L) Cd: 0,2-14,6;0,6-205; Al: 200-660.

Bidessus minutissimus (Germar, 1824)

(30.05.88) E11: 1.

Cd: 0,$4 ; \mathrm{CI}: 1,2 ; \mathrm{Al}: 360$.

Bidessus saucius ab coxalis (Sharp, 1880-82)

(30.05.88) E11: 1.

Cd: 0,$4 ; \mathrm{Cl}: 1,2 ; \mathrm{Al}: 360$.

Hydroglyphus pusillus (F., 1781)

(29.08.88) E1: 1.

Cd: 8,4; CI: 97,1; Al: 240.

Hydroporus (s. str.) basinotatus (Reiche, 1864)

(24.03.88) E6: 2, E8: 5, E1 1: 2; (30.05.88) E8: 83; (29.08.88)E8: 43, E9: 2; (31.10.88) E8: 58.

Cd: $0,2-1.1 ;$ Cl: $0,6-36,5 ; \mathrm{Al}: 140-660$.

Hydroporus (s. str.) lucasi (Reiche, 1866)

(24.03.88) E6: 17, E8: 1; (30.05.88) E8: 1: (29.08.88)E8: 1; (31.10.88) E3: 1, E8: 6.

Cd: 0,2-1,6; Cl: 0,7-36,5; Al: 120-660.

Hydroporus (s. str.) obsoletus (Aubé, 1836)

(30.05.88) E8: 3.

Cd: 0,$3 ; \mathrm{Cl}: 0,9 ; \mathrm{Al}: 660$.

Hydroporus (s.str.) tessellatus (Drapiez, 1819)

(24.03.88) E6: 1; (30.05.88) ES: 1.

Cd: 0,3-, 1; Cl: 0,9-36,5; Al: 140-660.

Hydroposurs sp (Clairville)

(24.03.88)E1: 4 (L); (30.05.88)EI: 6 (L), E2: 1 (L).

(L) Cd: 3,3-8,4; CI: 22,3-72; Al: 180-240.
Graptodytes ignotus (Mulsant, 1861)

(29.08.88) E8: 1 .

$\mathrm{Cd}: 0,5 ; \mathrm{Cl}: 0,7 ; \mathrm{Al}: 660$.

Graptodytes varius (Aubé, 1836)

(29.08.88)E8: 13; (31.10.88) El: 1, E8: 7

Cd: 0,5-8,6; CI: 0,7-107; Al: 240-260.

Graptodytes sp (Seidlitz)

(12.01.88) E6: 1 (L); (24.03.88)E6: 3 (L)

(L) Cd: 1,1-3,2; CI: 30,7-36,5; Al: 140.

Metaporus meridionalis (Aubé, 1836)

(12.01.88) E6: 1

Cd: 3,$2 ; \mathrm{Cl}: 30,7 ; \mathrm{Al}: 140$.

Scarodytes halensis ( $F ., 1787)$

(12.01.88) E5: 3 (L); (24.03.88) E5: 85 (L); (30.05.88)E5:

18 (L), E11: 1 .

(L) Cd: 9,8-14,6; Cl: 152-205; Al: 200.

Cd: 0,4; CI: 1,2; AI: 360 .

Stictonectes optatus (Seidlitz, 1887)

(29.08.88)E1: 2, E8: 1.

Cd: $0,5-8,5 ; \mathrm{Cl}: 0,7-97,1 ;$ Al: $240-660$

Deronectes fairmairei (Leprieur. 1876)

(12.01.88) E1: 1, E5: 9 (L) 2; (24.03.88) E5: 13 (L); (30.05.88)E1: 23, E2: 6, E3: 1, E5: 4 (L),E8: 1, E9: 1, E1 1: 8; (29.08.88) E1: 1 (L) 1, ES: I (L);(31.10.88) E9: 2; (19.01.89) E9: 1

(L)Cd: 8.4-14.6; CI: 97. 1-205; Al: 200-240.

Cd: 0,3-9,8; Cl: 0,7-152; Al: 120-660.

Deronectes hispanicus (Rosenhauer, 1856)

(30.05.88) ES: 1; (29.08.88)E8: 1.

Cd: 0,3-0,5; 0,7-0,9; Al: 660 .

Deronectes opatrinus (Germar, 1824)

(30.05.88)El 1: 2.

Cd: 0,4; Cl: 1,2; Al: 360.

Potamonectes (s.str.) cerisy (Aubé, 1836)

(12.01.88) E5: 33; (24.03.88) E5: 18; (30.05.88) E1: 6, E5:

36; (29.08.88) E1: 2, E5: 223.

Cd: 3,6-14,6; Cl: 72-205; Al: 200-240.

Potamonectes (s. str.) clarki (Wollaston, 1862) 
(30.05.88) E1 1: 2; (31.10.88) E2: 1, E9: 1; (19.01.89)E9: 1. Cd: 0,4-3,1; Cl: 0,7-17,9; Al: 180-380.

Potamonectes (s. str.) sansi (Aubé, 1836)

(29.08.88) E9: 1 .

Cd: 0,9; Cl: 0,6; Al: 380 .

Laccophilus hyalinus (De Geer, 1774)

(24.03.88) E1: 1 (1); (30.05.88) E1: 2 (L), E9: 4 (L) 2; (29.08.88)E1: 19 (L) 1, E2: 1 (L), E9: 6 (L); (19.01.89) E8: 1 (L).

(L) Cd: 0,6-8,4; Cl: 0,6-97, 1; Al: 180-660.

Cd: 0,6-8,4; CI: 0,9-97,1; Al: 240-380.

Agabus (Gabinectes) brunneus (F., 1798)

(29.08.88)E8: 1; (31.10.88)E1: 1.

Cd: 0,5-8,6; Cl: 0,7-107; Al: 240-660.

Agabus (Gabinectes) didymus (Olivier, 1795)

(24.03.88) E7: 1; (31.10.88) E3: 1.

Cd: 0,3-1,6; Cl: 0,8-9; Al: 70-120.

Agabus (Dichoaectes) biguttatus (Olivier, 1795)

(30.05.88) E8: 7; (31.10.88) E8: 1.

Cd: 0,3-0,5; Cl: 0,7-0,9; Al: 660 .

Agabus (Gaurodytes nebulosus (Forster, 1771)

(24.03.88) E3: 1 .

Cd: 6,2; Cl: 15,4; Al: 120.

Agabus sp (Leach)

(12.01.88) E1: 1 (L), E6: 1 (L); (24.03.88) E6: 2 (L); (30.05.88) E1: 1 (L); (31.10.88) E1: 7 (L); (19.01.89) E2: 1 (L).

(L) Cd: 1, 1-8,6; Cl: 30,7-107; Al: 140-240

Ilybius sp (Erichson)

(24.03.88) E6: 2 (L).

(L) Cd: 1,1; CI: 36,5; Al: 140.

Colymbetes fuscus (L., 1758)

(12.01.88) E6: (L); (30.05.88)E3: 1.

(L) Cd: 3,2; Cl: 30,7; Al: 140 .

Cd: 3,0; Cl: 22,1; A: 120 .

Meladema coriacea (Castenau, 1834)

(24.03.88) El 1: 2 (L).

(L) Cd: 0,2; CI: 1.2; Al: 360 .
Hydaticus leander (Rossi, 1979) Leach

(19.01.89)E9: 3 (L).

(L) Cd: 0,7; Cl: 0,7; Al: 380 .

Hydraena (Phothydraena) hernandoi (Fresneda \& Lagar, 1990)

(29.08.88)E8: 1.

Cd: 0,5; Cl: 0,7; Al: 660 .

Hydraena (s.str.) andalusa (Lagar \& Fresneda. 1990) (30.05.88)E8: 6; (29.08.88)E8: 4; (19.01.89) E8: 3.

Cd: 0,3-0,8; Cl: 0,7-2: Al: 660 .

Hydraena (s.str) capta (Orchyinont, 1936)

(30.05.88) E8: 21, E1 1: 1; (29.08.88) E8: 15, E9: 2: (31.10.88) E8: 3.

Cd: 0,3-0,9; Cl: 0,6-1,2; Al: 360-660.

Hydraena (s. str.) cordata cordata (Schaufuss, 1883) (30.05.88) E8: 1, E11: 3; (29.08.88): E8: 3; (19.01.89) E8: 1. Cd: 0,3-0.8; Cl: 0,7-2,1; Al: 360-660.

Hydraena (s.str.) subdepressa (Rey, 1886)

(12.01.88) E1: 2; (30.05.88)E1: 1; (29.08.88) E8: 2, E9: 1.

Cd: 0,5-4,1; Cl: 0,6-72; Al: 240-660.

Hydraena (Haenydra) gaditana (Lagar \& Fresneda, 1990) (24.03.88) E8: 7; (30.05.88) E8: 17; (29.08.88) E8: 1; (31.10.88)E8: 3 ; (19.01.89)E8: 1.

Cd: $0,2-0,8 ; \mathrm{Cl}: 0,7-2,1 ; 660$.

Ochthebius (Asiobates) bonnairei (Guillebeau, 1896)

(30.05.88)E9: 1, El 1: 1.

Cd: 0,4-0,6; CI: 0,9-1,2; Al: 360-380.

Ochthebius (Asiobates) dilatatus (Stephens, 1829)

(24.03.88) E5: 11, E7: 4, E10: 3; (30.05.88) E5: 3;

(31.10.88)E1: 1

Cd: 0,3-14,6; Cl: 0,8-205; Al: 70-240.

Ochtebius (Hymenodes) quadrifossulatus (Walt)

(30.05.88)E5: 1; (29.08.88)E1: 3, E5: 1

Cd: 8,4-10,1; CI: 97,1-191; Al: 200-240.

Ochthebius (Bothochius) punctatus (Stephens, 1829).

(12.01.88) E5: 4

Cd: 9,8; CI: 152; Al: 200.

Ochtebius (s.str.) viridis (Peyron, 1859)

(31.10.88)El; 3 .

Cd: 8,6; Cl: 107; Al: 240. 
Hydrochus sp (Leach)

(30.05.88) El 1: 1

Cd: 0,$4 ; \mathrm{Cl}: 1.2 ; \mathrm{Al}: 360$.

Helophorus sp. F.

(12.01.88)E6: 1; (24.03.88)E7: 1; (31.10.88) E8: 1

Cd: 0,3-3,2; Cl: 0,7-30,7; Al: 70-660.

Berosus hispanicus (Küster, 1841)

(12.01.88) E5: 14 (L) 6; (24.03.88)E5: 13 (L);(30.05.88)El :

14 (L), E5: 38 (L) 8; (29.08.88)El 39 (L) 371, E5 (L) 330;

(31.10.88) E1: 25 (L) 14, E3: 5; (19.01.89) E1: 11 (L) 3.

(L) Cd: 3,6; Cl: 72-205; Al: 200-240.

Cd: 1,6-10,1; Cl: 9,0-191; Al: 120-240.

Paracymus aeneus (Germar)

(30.05.88) E8: 1.

Cd: 0,3; Cl: 0,9; Al: 680.

Anacaena globulus (Paykull, 1798)

(30.05.88) E8: 1 .

Cd: 0,3; Cl: 0,9; Al: 660 .

Laccobius atratus (Rottenberg, 1874).

(24.03.88) El 1: 1; (29.08.88) E1: 26, E5: 3.

Cd: 0,2-10,1; CI: 1,2-137; Al: 200-360.

Laccobius atrocephalus (Reitter, 1872)

(30.05.88) E2: 1, E3: 6, E10: 1, E11: 1, (29.08.88) E1: 39, E2: 1; (31.10.88)E3: 3; (19.01.89)E1: 2.

Cd: 0,4-8,4; Cl: 1,2,97. 1; Al: 120-360.

Laccobius sp Erichson

(24.03.88) E5: 2 (L); (30.05.88) E1: 33 (L), E3: 19 (L), E5:

10 (L), E10: 2 (L), E1 1: 7 (L); (29.08.88)E1: 29 (L), E4: 3

(L), E9: 2 (L); (19.01.89) E3: 2 (L).

(L) Cd: 0,4-14,6; Cl: 0,6-205; Al: 80-380.

Enochrus Morenae Heyd

(29.08.88)E1: 1, E5: 1 (L); (31.10.88)El: 1

(L) Cd: 10.1; CI: 137; Al: 200.

Cd: 8,4-8,6; Cl: 97,1-107; Al: 240.

Limnebius fretalis (Peyerimhoff, 1912)

(30.05.88) E8: 1; (19.01.89)E8: 2.

Cd: 0,3-0,8: Cl: 0,9-2,1; Al: 660 .

Dryops sp Olivier

(24.03.88)E1 1: 1 (L); (30.05.88) E10: 1, El 1: 1; (29.08.88)

E8: 1, E9: 7 (L).

(L) Cd: $0,2-0,9 ; \mathrm{Cl}: 0,6-1,2 ; \mathrm{Al}: 360-380$.

Cd: 0,4-1,3; Cl: 0,7-4,5; Al: 240-660.

Dupophilus brevis Mulsant \& Rey, 1872
(19.01.89) E8: 1 (L).

(L)Cd: 0,$8 ; \mathrm{Cl}: 2,1 ; \mathrm{Al}: 660$

Elmis maugetil Latreille, 1798

(24.03.99) E8: 20 (L) 9, E9; 1 (L); (30.05.88) E8: 9 (L) 10; (29.08.88)E8: 1 (L) 2, E9: 1 (L); (31.10.88)E8: 6 (L) 1, E9:

1 (L); (19.01.89) E8: 14 (L) 3, E9: 4 (L).

(L) Cd: $0,2-0,9 ;$ CI: 0,6-2,1; Al: 380-660.

Cd: 0,2-0,8; Cl: 0,7-2,1; Al: 660 .

Esolus sp Mulsant \& Rey

(12.01.88) E9: 1 (L); (24.03.88) E9: 1 (L): E10: 4 (L); (29.08.88) E10: 1 (L)

(L) Cd: $0.5-1,2 ;$ CI: $1,0-5,1 ; \mathrm{Al}: 240-380$.

Limnius sp Illiger

(24.03.88) E8: 5 (L), E9: 3 (L) 1; (30.05.88) E8: 1 (L) 5; (29.08.88)E8: 1 (L),E9: 1 (L) 1; (31.10.88) E8: 5 (L), E9: 4 (L); (19.01.89) E9: 4 (L) 1

(L) Cd: 0,2-0,9; CI: 0,6-1,0; Al: 380-660.

Cd: $0,3-0,9 \mathrm{Cl}: 0,6-1,0 ; \mathrm{Al}: 380-660$.

Oulimnius rivularis (Rosenhauer, 1856)

(12.01.88)E1: 2; (24.03.88)E8: 1 (L), E9: 1 (L), E10: 15 (L), El 1: 2 (L) 3; (30.05.88) E1: 35 (L), E2: 1 (L), E8: 2, E1 1: 20

(L) 1; (29.08.88) E9: 15 (L) 1, E10: 2 (L); (31.10.88) E8: 1

(L). E9: 5 (L); (19.01.89) E8: 13 (L), E9: 8 (L).

(L) Cd: 0,2-3,6; Cl: 0,6-72; Al: 180-660.

Cd: 0,2-4,1; CI: 0,6-38,7; Al: 240-660.

Riolus subviolaceus (Müller, 1817)

24.03.88) E8: 154 (L) 16, E9: 1 (L); (30.05.88) E8: 9 (L) 31; (29.08.88) E8: 2 (L) 6; (31.10.88)E8: 3; (19.01-89) E8: 11 (L) 4, E9: 1

(L) Cd: 0,2-0,8; Cl: 0,7-2.1; Al: 660 .

Cd: 0,2-0,8; CI: 0,7-2,0 Al: 660 .

Cd: 0,2-0,8; Cl: 0,7-2,1; Al: 380-660

Stenelmis sp Dufour

$(31.10,88) \mathrm{E} 8: 1(\mathrm{~L})$.

(L) Cd: 0,$5 ; \mathrm{CI}: 0,7 ; \mathrm{Al}: 660$

Helodes $\mathrm{sp}$

(31.10.88)E8: 3 (L); (19.01.89) E8: 6 (L).

(L) Cd: 0,5-0,8; CI: 0,7-2,1; Al: 660.

Hydrocyphon $\mathrm{sp}$

(24.03.88) E8: 7 (L); (30.05.88) E8: 4 (L); (19.01.89) E8: 1 (L).

(L) Cd: 0,2-0,8; CI: 0,7-2,1; Al: 660. 
Con el fin de comprobar el patrón de distribución de los taxa recogidos en ambas cabeceras, se ha usado el Índice de Representación (I.R.) (HILDREW \& TOWNSEND, 1976), utilizando la terminología propuesta por CUPPEN (1986): los valores positivos del índice indican preferencia y los negativos indican aversión. Las diferencias cn los valores del índice son consideradas significativas cuando el valor se desvía dos o más de cero (TOLKAMP, 1980). Los valores del índice de Representación para los taxa presentes en al menos el 10 por ciento dc las muestras con respecto a cloruro se dan en la Tabla I se obtuvieron valores significativos para todos los taxa. Aquellos que mostraron preferencia hacia aguas con muy bajo contenido en cloruro fueron $G$. dejeani, H. basinotatus, E. rnaugetii (larvas), y $R$. subviolaceus (larvas y adultos). H. capta, $\mathrm{H}$. gaditana, E. maugetii (adultos) y Limnius sp también mostraron esta preferencia, pero sin tener aversión significativa hacia los valores más altos. Los taxa que mostraron preferencia hacia aguas con alto contenido en cloruro fueron Y. bicarinata, D. fairmairei (especialmente en estadío larvario), P. cerisyi, B. hispanicus (larvas y adultos), Agabus sp, L. atrocephalus, y Laccobius sp (larvas). Las especies de Ochthebius mostraron, en general, preferencia hacia aguas muy salinas, aunque existió una segregación hacia distintos niveles de salinidad. $O$. bonnairei se encontró cn las aguas menos salinas del Guadalete: Grazalema y Águila, O. quadrifossulatus en las más saladas del Salado y Barros, y O. punctatus y $O$. viridis en un rango más amplio de salinidades, pero siempre cn la cabecera de la cuenca del Guadaira. Otras especies como H. lineatocollis, L. hyalinus у 0 . dilatatus mostraron preferencia hacia aguas salinas, pero sin aversión, al mismo tiempo, hacia las menos salinas.

Los valores del índice de Representación para los taxa presentes en al menos el 10 por ciento de las muestras con respecto a la altitud se dan en la Tabla II. Igual que para cloruro se encontraron valores significativos para todos los taxa. C. deieani, H. basinotatus, $H$. capta, E. maugetii (larvas) y $R$. subviolaceus (larvas y adultos) mostraron preferencia hacia las altitudes mayores. Otros taxa corno H. lineatocollis, H. gaditana, E. maugetii (adultos) y Limnius sp, también mostraron preferencia hacia altitudes altas, pero sin mostrar aversión significativa hacia las más bajas. O. rivularis fue el único Elmidae encontrado en los cstadíos de larva y adulto en las dos cuencas, aunque como larva mostró preferencia por las estaciones más altas del Guadalete. P. cerisyi y B. hispanicus (larvas y adultos) mostraron los valores más altos en las clases de altitud coincidentes con las estaciones de mayor salinidad (Barros y Salado). El mismo comportamiento, aunque menos acentuado, mostraron $Y$. bicarinata, D. fairmairei (larvas), O. dilatatus, Ochthebius $\mathrm{sp}, L$. atrocephalus y Laccobius sp (larvas). El resto de los laxa se mostraron más indiferentes a la altitud.
Al comparar los porcentajes de abundancia de los taxa dominantes con respecto al total de abundancia de la comunidad de Coleóptera obtenidos por ORTEGA et al. (1991) en la Rambla del Moro (SE de España) con los obtenidos en Barros (dos arroyos de igual salinidad) (Tabla III), se observa como Berosus sp B. hispanicus en Barros) fue el taxa dominante en ambos arroyos, junto con P. cerisyi (más abundante en Barros) y Ochthebius sp ( 0 . dilatatus, 0 . punctatus y O.quadrifossulatus en Barros). En la Rambla del Moro fue importante la abundancia dc Enochrus sp mientras que cn Barros la presencia de E. morenae fue testimonial, por el contrario $\mathrm{S}$. halensis fue el tercer taxa en abundancia en Barros, mientras que en la Rambla del Moro no se encontró.

Con el fin de discernir la influencia que la temporalidad ejerce sobre los taxa que habitan las dos cabeceras estudiadas, se ha construido la Tabla IV. En ésta se representan, en porcentajes, los valores de abundancia dc los taxa presentes en al menos el 10 por ciento de las muestras, en las clases en que se han ordenado los cauces según su temporalidad o no a lo largo del año estudiado (temporales, semipermanentes y permanentes) independientemente de la cuenca a que pertenezcan. G. dejeani, H. basinotatus, H. capta, H. gaditana, E. maugetii y $R$. subviolaceus, todos en estadío adulto, tuvieron una preferencia significativa por medios permanentes (test chicuadrado $\mathrm{p}<0.001)$. E. maugetii, Limnius sp y $R$. subviolaceus $(\mathrm{p}<0.001)$ y $O$. rivularis $(\mathrm{p}<0.05)$, todos cn cstadío larvario, mostraron la misma preferencia. Y. bicarinata, D. fairmairei, $B$. hispanicus y Laccobius $\mathrm{sp}(\mathrm{p}<0.001)$, todos como larvas, preferían medios temporales o semipcrmanentes, al igual que $\mathrm{P}$. cerisyi, Ochthebius sp, B. hispanicus y L. atrocephalus $(p<0.001)$ en estadío adulto. El resto de los taxa no mostraron ninguna preferencia significativa con algún tipo de medio.

\section{DISCUSIÓN}

Las preferencias encontradas para $G$. dejeani, H. basinotatus, H. capta, H. gaditana, Elmis maugetii (preferentemente en estadío larvario), Limnius sp y $R$ subviolaceus por aguas con muy baja salinidad y altitudes altas, posiblemente se correspondan, en realidad, con una tendencia hacia aguas con corricnte durante todo el año, ya que en el área de estudio se da esta relación, de manera que las altitudes mayores (clases 7 y 8 del I. R.) pertenecían a cauces con corriente todo el año y niveles muy bajos de salinidad (Río Guadalete en Grazalema y Gaidovar). Estas especies, además,mostraron preferencias significativas por cauces de aguas permanentes (Tabla IV).

Sin embargo, las especies que mostraron preferencias por las aguas más salinas (Y. bicarinata, D. fairmairei (larvas), $P$. 


\begin{tabular}{|c|c|c|c|c|c|c|c|c|}
\hline & $<3,0$ & $3,1-5,5$ & $5,6-10,1$ & $10,2-18$ & $18,6-33$ & $33,8-61$ & $61,6-11$ & $>112$ \\
\hline H. lineatocollis 1 & 0,62 & $-1,35$ & $-1,13$ & $-1,55$ & $-1,43$ & 1,13 & 1,89 & 4,08 \\
\hline G. dejeani & 15,67 & $-4,65$ & $-3,87$ & $-5,32$ & $-4,91$ & $-3,87$ & $-3,53$ & $-4,47$ \\
\hline Y. bicarinata 1 & $-2,31$ & $-2,49$ & $-2,07$ & $-2,85$ & $-2,63$ & $-1,59$ & 1,28 & 15,56 \\
\hline H. basinotatus & 14,81 & $-4,46$ & $-3,71$ & $-5,11$ & $-4,71$ & $-3,17$ & $-3,39$ & $-4,28$ \\
\hline H. lucasi & 1,75 & $-1,97$ & $-1,03$ & $-2,25$ & $-2,08$ & 8,74 & $-1,49$ & $-1,89$ \\
\hline D. fairmairei 1 & $-3,16$ & $-1,69$ & $-1,41$ & $-1,93$ & $-1,79$ & $-1,41$ & $-0,51$ & 14,99 \\
\hline D. fairmairei & $-0,92$ & $-2,19$ & $-1,82$ & $-2,51$ & 0,71 & $-1,27$ & 12,76 & $-1,15$ \\
\hline P. cerisyi & $-10,65$ & $-5,69$ & $-4,74$ & $-6,51$ & $-6,01$ & $-4,74$ & $-2,47$ & 51,19 \\
\hline L. hyalinus 1 & $-0,33$ & $-1,86$ & $-1,55$ & $-2,13$ & $-1,46$ & $-0,91$ & 13,42 & $-1,79$ \\
\hline Agabus sp 1 & $-2,07$ & $-1,11$ & $-0,92$ & $-1,26$ & $-0,31$ & 2,34 & 8,68 & $-1,06$ \\
\hline H. capta & 6,99 & $-2,07$ & $-1,72$ & $-2,36$ & $-2,18$ & $-1,72$ & $-1,57$ & $-1,99$ \\
\hline H. gaditana & 5,81 & $-1,72$ & $-1,43$ & $-1,96$ & $-1,81$ & $-1,43$ & $-1,31$ & $-1,65$ \\
\hline O. dilatatus & $-1,38$ & 0,51 & $-1,25$ & $-1,71$ & $-1,58$ & $-1,25$ & $-0,26$ & 8,27 \\
\hline Ochthebius sp & $-6,06$ & $-3,96$ & 14,29 & $-4,09$ & $-3,71$ & $-2,99$ & $-0,02$ & 15,11 \\
\hline B. hispanicus 1 & $-7,27$ & $-3,88$ & $-3,23$ & $-4,44$ & $-4,11$ & $-3,23$ & 23,48 & 15,02 \\
\hline B. hispanicus & $-16,18$ & $-8,65$ & $-6,51$ & $-9,89$ & $-9,14$ & $-6,51$ & 52,02 & 33,07 \\
\hline L. atrocephalus & $-4,08$ & $-1,87$ & $-0,35$ & $-2,63$ & 0,86 & $-1,92$ & 20,55 & $-2,21$ \\
\hline Laccobius sp 1 & $-4,72$ & $-2,69$ & $-1,66$ & $-3,78$ & 1,96 & $-2,75$ & 22,21 & 0,61 \\
\hline E. maugetii 1 & 8,13 & $-2,41$ & $-2,01$ & $-2,76$ & $-2,55$ & $-2,01$ & $-1,83$ & $-2,32$ \\
\hline E. maugetii & 5,39 & $-1,59$ & $-1,33$ & $-1,83$ & $-1,69$ & $-1,33$ & $-1,21$ & $-1,53$ \\
\hline Limnius sp 1 & 5,28 & $-1,56$ & $-1,31$ & $-1,79$ & $-1,65$ & $-1,31$ & $-1,19$ & $-1,51$ \\
\hline O. rivularis 1 & 6,22 & $-3,48$ & $-2,91$ & $-3,98$ & $-3,41$ & $-2,91$ & 10,59 & $-3,35$ \\
\hline O. rivularis & 2,09 & $-0,96$ & $-0,79$ & $-1,11$ & $-1,01$ & 1,71 & $-0,73$ & $-0,92$ \\
\hline R. subviolaceus 1 & 14,33 & $-4,25$ & $-3,53$ & $-4,86$ & $-4,49$ & $-3,53$ & $-3,23$ & $-4,08$ \\
\hline R. subviolaceus & 8,42 & $-2,49$ & -2.07 & $-2,85$ & $-2,63$ & $-2,07$ & $-1,89$ & $-2,39$ \\
\hline
\end{tabular}


TABLA II. Valores del índice de Representación para los taxa presentes al menos en el 10 por ciento de las muestras, con respecto a la altitud. (1= larvas) TABLE II. Index of representation values for taxa of Coleoptera, present at least in the 10 por cent of the samples, with respect to altitude. (1= larves).

\begin{tabular}{lrrrrrrrr}
\hline & $<93$ & $94-123$ & $124-163$ & $164-215$ & $216-285$ & $286-378$ & $379-500$ & $>500$ \\
\hline H. lineatocollis 1 & $-1,53$ & $-1,46$ & $-0,83$ & 1,34 & $-0,02$ & $-1,05$ & 4,03 & $-1,53$ \\
G. dejeani & $-5,11$ & $-4,87$ & $-2,77$ & $-6,89$ & $-6,72$ & $-3,49$ & 29,01 & 4,11 \\
Y. bicarinata 1 & $-2,73$ & $-2,61$ & $-1,48$ & 7,95 & $-1,66$ & 1,32 & $-1,08$ & $-2,37$ \\
H. basinotatus & $-4,89$ & $-4,67$ & $-1,91$ & $-6,61$ & $-6,44$ & $-2,76$ & $-4,24$ & 33,74 \\
H. lucasi & $-2,16$ & $-1,57$ & 13,36 & $-2,91$ & $-2,84$ & $-1,48$ & $-2,06$ & 7,11 \\
D. fairmairei 1 & $-1,85$ & $-1,77$ & $-1,01$ & 8,29 & $-2,03$ & $-1,27$ & $-1,77$ & $-1,85$ \\
D. fairmairei & $-2,39$ & $-1,86$ & $-1,31$ & $-0,77$ & 4,74 & 3,21 & $-0,55$ & $-1,98$ \\
P. cerisyi & $-6,25$ & $-5,96$ & $-3,39$ & 28,31 & $-7,26$ & $-4,29$ & $-5,96$ & $-6,25$ \\
L. hyalinus 1 & $-2,04$ & $-1,95$ & $-1,11$ & $-2,39$ & 5,49 & $-1,39$ & 3,18 & $-1,55$ \\
Agabus sp 1 & $-1,26$ & $-1,21$ & 3,69 & $-1,12$ & 3,74 & $-0,87$ & $-1,21$ & $-1,26$ \\
H. capta & $-2,27$ & $-2,17$ & $-1,23$ & $-3,06$ & $-2,99$ & $-0,91$ & $-1,24$ & 14,91 \\
H. gaditana & $-1,88$ & $-1,79$ & $-1,02$ & $-2,55$ & $-2,48$ & $-1,29$ & $-1,79$ & 13,49 \\
O. dilatatus & 0,79 & $-1,57$ & $-0,89$ & 4,09 & $-0,32$ & $-1,13$ & $-1,57$ & $-1,64$ \\
Ochthebius sp & $-3,77$ & 10,84 & $-2,41$ & 6,16 & $-3,63$ & $-1,74$ & $-4,01$ & $-3,77$ \\
B. hispanicus 1 & $-4,42$ & $-4,22$ & $-2,39$ & 5,77 & 9,47 & $-3,03$ & $-4,22$ & $-4,42$ \\
B. hispanicus & $-9,51$ & $-8,53$ & $-5,16$ & 13,94 & 18,44 & $-6,52$ & $-9,08$ & $-9,51$ \\
L. atrocephalus & $-2,57$ & 1,19 & $-1,41$ & $-2,89$ & 8,99 & $-1,19$ & $-2,46$ & $-2,57$ \\
Laccobius sp 1 & $-2,84$ & 2,52 & $-1,98$ & $-2,51$ & 8,46 & 0,28 & $-2,92$ & $-3,66$ \\
E. maugetii 1 & $-2,65$ & $-2,53$ & $-1,44$ & $-3,57$ & $-3,49$ & $-1,82$ & 0,24 & 16,24 \\
E. maugetii & $-1,75$ & $-1,67$ & $-0,95$ & $-2,37$ & $-2,31$ & $-1,19$ & $-1,67$ & 12,49 \\
Limnius sp 1 & $-1,68$ & $-1,59$ & $-0,91$ & $-2,27$ & $-2,21$ & $-1,15$ & 5,88 & 4,87 \\
O. rivularis 1 & $-3,82$ & $-3,65$ & $-2,07$ & $-4,97$ & 5,29 & 5,77 & 4,31 & 0,09 \\
O. rivularis & $-1,05$ & $-0,99$ & $-0,57$ & $-1,42$ & 0,06 & 4,83 & $-0,01$ & 0,85 \\
R. subviolaceus 1 & $-4,66$ & $-4,45$ & $-2,53$ & $-6,29$ & $-6,14$ & $-3,21$ & $-4,23$ & $-33,09$ \\
R. subviolaceus & $-2,74$ & $-2,61$ & $-1,49$ & $-3,69$ & $-3,59$ & $-1,88$ & $-2,23$ & 19,24 \\
\hline & & & & & & & &
\end{tabular}


TABLA III. Comparación de los taxa mayoritarios de Coleoptera presentes en la Rambla del Moro (ORTEGA et al., 1991) y Arroyo de Barros. Valores expresados en porcentajes; conductividad en $\mathrm{mS} . \mathrm{cm}^{1}$.

TABLE III. Comparison of the more important taxa of Coleopera present in Rambla del Moro (ORTEGA ef al., 1991) and Barros stream. Values in per cent; conductivity in $\mathrm{mS} . \mathrm{cm}$ ?

\begin{tabular}{lrr}
\hline & R. del Moro & \multicolumn{1}{c}{ Barros } \\
\hline Rango de conductividad & $9,2-13,5$ & $9,8-14,6$ \\
P. cerisyi & 19,0 & 30,5 \\
Ochthebius sp & 10,6 & 8,3 \\
Berosus sp & 46,0 & 40,7 \\
Enochrus sp & 18,8 & 0,1 \\
S. halensis & - & 10,4 \\
Total & 94,4 & 90,0 \\
\hline
\end{tabular}

TABLA IV. Distribución de los taxa de Coleoptera, presentes en al menos el 10 por ciento de las muestras, según la permanencia del agua en el medio. Tempor: aguas temporales (se secan totalmente durante el estiaje); Semipe: aguas semipermanente (durante el estiaje quedan pozas, en la mayor parte de los casos sin solución de continuidad); Perman: aguas permanentes (agua con corriente durante todo el año). $1=$ ejemplares en estadío larvario. (test de chi-cuadrado con bondad de ajuste: $* \mathrm{p}<0.05 ; * * \mathrm{p}<0.001 ; * * * \mathrm{p}=0 ; 1 \mathrm{~g} .1$.)

TABLE IV. Coleoptera distribution according to water persistence, only taxa present at least in the 10 per cent of the samples. Tempor: temporary streams (completely dried during the drought period); Semipe: streams made of by isolated pools during the drought period; Perman: streams with flow throughout the year $/ / 1=$ larves. (Chi square with good fitness: ${ }^{*} p<0.05 ;{ }^{* *} \mathrm{p}<0.001 ;{ }^{* * *} \mathrm{p}=0 ; 1 \mathrm{~d}$. f.)

\begin{tabular}{|c|c|c|c|}
\hline & Tempor. & Semipe & Perman. \\
\hline H. lineatocollis (1) & 0,0 & 45,3 & 54,7 \\
\hline G. dejeani $* *^{*}$ & 0,0 & 0,0 & 100,0 \\
\hline Y. bicarinata $(1){ }^{* *}$ & 6,8 & 86,1 & 7,1 \\
\hline H. basirzotatus ${ }^{* *}$ & 1,9 & 0,0 & 98,1 \\
\hline H. lucasi & 48,4 & 0,0 & 51,6 \\
\hline D. fairmairei (1) *** & 0,0 & 100,0 & 0,0 \\
\hline D. fairmairei & 22,2 & 54,5 & 23,2 \\
\hline P. cerisyi $*^{*}$ & 0,0 & 100,0 & 0,0 \\
\hline L. hyalinus (1) & 0,0 & 57,4 & 42,6 \\
\hline Agabus sp (1) & 21,4 & 75,0 & 3,6 \\
\hline H. capta ${ }^{\star \star}$ & 3,6 & 0,0 & 96,4 \\
\hline H. gaditana $* *$ & 0,0 & 0,0 & 100,0 \\
\hline O. dilatatus & 19,4 & 65,7 & 14,9 \\
\hline Ochthebius sp ** & 47,6 & 50,2 & 2,2 \\
\hline B. hispanicus (1)** & 0,0 & 100,0 & 0,0 \\
\hline B. hispanicus ${ }^{\star * *}$ & 1,1 & 98,9 & 0,0 \\
\hline L. atrocephalus ** & 23,4 & 72,3 & 4,3 \\
\hline Laccobius $\operatorname{sp}(1) * *$ & 27,3 & 68,9 & 3,8 \\
\hline E. maugetii $(1) * *$ & 0,0 & 0,0 & 100,0 \\
\hline E. maugetii ${ }^{* *}$ & 0,0 & 0,0 & 100,0 \\
\hline Limnius $\operatorname{sp}(1) * *$ & 0,0 & 0,0 & 100,0 \\
\hline 0 .rivularis $(1)$ * & 21,1 & 28,2 & 50,7 \\
\hline 0. rivularis & 41,4 & 20,7 & 37,9 \\
\hline R. subviolaceus $(1){ }^{* *}$ & 0,0 & 0,0 & 100,0 \\
\hline R. subviolaceus $* *$ & 0,0 & 0,0 & 100,0 \\
\hline
\end{tabular}


cerisyi, Ochthebius sp, y Berosus hispanicus (larvas y adultos) se encontraron en las clases de altitud correspondientes a los cauces más salinos, por lo que parece que es este factor y no la altitud el que más intluyc cn sus distribuciones. Algunas de ellas (O. dilatatus, O. punctatus, O. viridis y P. cerisyi) han sido citadas como indicadoras de aguas salinas (IENISTEA, 1978; PIRISINU, 1981; MONTES \& RAMÍREZ, 1981; JAECH \& MARGALIT, 1987). Estas mismas especies, junto con $L$. atrocephalus y Laccobius sp, se encontraron preferentemente en aguas semipermanentes y/o temporales lo que unido, al hecho de su preferencia por los medios muy salinos (que no pueden ser explotados por un gran número de especies), parece demostrar que poseen un carácter oportunista muy desarrollado.

La comunidad encontrada en la cuenca del Guadaira inostró una gran similitud con la de la Rambla del Moro (Ortega et al., 1991) (Tabla III), constituidas ambas por P. cerisvi y B. hispanicus (Berosus sp en la Rambla) y un pequeño grupo de especies acompañantes variables. Esta comunidad podría ser representativa de cauces con una fuerte irregularidad eri su caudal y largo período de estiaje, que a su vez poseen una alta mineralización.

\section{BIBLIOGRAFÍA}

BAENA. M. 1983. Hesperocorixa furtiva (Horváth, 1907) (Het. Corixidae) nueva para la Península Ibérica. Bol. Asoc. esp. Entom., 7: 319.

BAENA, M. 1985. Una nueva especie de Parasigara del Sur de España: Parasigara baetica n. sp (Heteroptera, Corixidae). Nouv. Revue Ent. (N. S.), 2 (1): 107-110.

BERTOLET, J.. M.J. PORTILLO, A. REYES, P. MUÑOZ, J. PALACIOS \& A. GARCÍA, 1989. Cuadernos de Jerez. El Guadalete. Ed. Del. Educación y Cultura. Ayuntamiento de Jcrez.

BURMEISTER, E.G. 1983. Agabus (Gaurodytes) hozgargantae sp. nov. aus Südspanien (Coleoptera, Dytiscidae). Spixiana, 6 (2): 133-139.

CUPPEN, J.G.M. 1986. The influence of acidity and chlorinity on the distribution of Hydroporus species (Coleoptera. Dytiscidae) in the Netherlands. Entomologica Basiliensia, 11: 327-336.

DIRECCIÓN GENERAL OBRAS HIDRÁULICAS, 1979 1991. Análisis de calidad de agua. Publ. M.O.P.T. Madrid.

FERY, H. \& J. FRESNEDA, 1988. Deronectes algibensis $n$. sp. vom aüssersten Süden Spaniens (Coleoptera: Dytiscidae). Ent. Z., 98 (23): 337-352.

GALLARDO, A. 1991. Respuesta de macroinvertebrados fluviales a la salinidad. Comparación de las cuencas de los rios Guadaira y Guadalete. Tesis Doctorales en microfichas n. 33 (1993). Ed. Universidad de Sevilla.
GALLARDO, A. 1993. Macroinvertebratc associations in two basins of SW Spain. Archiv Hydrobiol, 127-483.

GALLARDO, A. \& J. PRENDA, 1994. Influence of some enviroiimental, factors on the freshawater macroinvertebrates distribution in two adjocent river basins incler mediterranean climate. J. Dipteran larvae (excepting chironominds and simulidae a escological indicator. Archiv. Hychobiol., 131: 435-447.

GALLARDO, A. \& J. TOJA. 1989. Efecto de la contaminación orgánica en los macroinvertebrados acuáticos en la Cuenca del Río Guadaira (Sevilla, SW España). Actas Col. Luso-Esp. Ecol. Bacias Hidrogr: e Rec. Zool: 163-170.

HERNANDO, C. \& J. FRESNEDA, 1987. Contribució al coneixement dels Hydradephaga (Col. Dytiscidae i Gyrinidade) de la Península Ibérica. Butll. de la U.E.C., 149: 312-315.

HILDREW, A.G. \& C.R. TOWNSEND, 1976. The distribution of two predators and their prcy in an iron rich strearn. .1. Anim. Ecol., 45: 41-57.

IENISTEA, M.A. 1978. Hydratephaga und Palpicornia. En: Limnofauna Europaea (J. ILLIES. ed.) 291-314. Gustav Fischer Verlag. Stuttgart.

JAECH, M.A. \& J. MARGALIT. 1987. Distribution of Dytiscids in springs of the Western Dead Sea Area (Coleoptera: Dytiscidae). The Coleopterists Bull., 41: 327-334.

LAGAR, A. \& J. FRESNEDA, 1990. Notas faunísticas y taxonómicas sobre Hydraenidac (Colcoptcra, Palpicornia) de la Península Ibérica: descripción de nucvas Hydraena ibéricas. Entornologie, 60: 149-160.

MONTES, C. \& L. RAMÍREZ, 1981. Indicadores ecológicos de algunos ecosistemas acuáticos del Rajo Guadalquivir (SW; España): Odonatos, heterópteros y coleóptcros acuáticos. Actas 1." Congreso Español de Limnología: 43-49.

ORTEGA, M., M.L. SUÁREZ, M.R. VIDAL-ABARCA \& L. RAMÍREZ-DÍAZ, 1991. Aspectos dinámicos de la composición y estructura dc la comunidad de invertebrados acuáticos de la Rambla del Moro después de una riada (Cuenca del Río Segura: SE de España). Limnética, 7: 11-24.

PIRISINU, Q. 1981. Guide per il riconoscimiento delle specte animali delle acque interne italiane. Vol. 13. Palpicorni Consiglio Nazionale delle Ricerche. Italy.

PRENDA, J. \& A. GALLARDO, 1992. The influence of environmental factors and rnicrohabitat availability on the distribution of an aquactic Oligochaeta assemblage in a Mediterraneam river basin. Int Revue. ges. Hydrobiol., 77: 421-434.

TOLKAMP, H.H. 1980. Organism-substrate relationships in lowland streams. Thesis, Centre for Agricultura1 Pub. \& Documentantion. Wageningen. 